\title{
Obstructive Sleep Apnea, Pain, and Opioid Analgesia in the Postoperative Patient
}

\author{
Anthony G. Doufas
}

Published online: 18 December 2013

(c) Springer Science + Business Media New York 2013

\begin{abstract}
Obstructive sleep apnea (OSA) is a common health problem among surgical patients. Human evidence supports that important components of OSA like sleep fragmentation and intermittent hypoxia may enhance pain behavior and also increase sensitivity to opioid analgesia. To the extent that these effects might affect postoperative opioid pharmacology, OSA may impact the risk for opioidinduced ventilatory impairment (OIVI), a potentially serious complication in the postoperative patient. On the other hand, certain pathophysiological features of OSA might promote the development of OIVI due to enhancing respiratory compromise and/or through suppressing arousal from sleep in response to an airway obstruction event. Nonetheless, possible determinants of OIVI are not limited to factors associated with sleep-disordered breathing and current evidence does not support a direct relationship between an isolated preoperative diagnosis of OSA and increased risk for OIVI during postoperative analgesic therapy. Older age, comorbidity burden, and increased postoperative sedation, seem to be important promoters of potentially severe OIVI in the postoperative patient. Accepted strategies to prevent OIVI without interfering with postoperative analgesia include adopting opioidsparing analgesic techniques, as well as establishing
\end{abstract}

\footnotetext{
A. G. Doufas $(\square)$

Department of Anesthesiology, Perioperative and Pain Medicine, Stanford University School of Medicine, 300 Pasteur Drive, Stanford, CA 94305, USA

e-mail: agdoufas@stanford.edu

A. G. Doufas

Outcomes Research Consortium, Cleveland, OH, USA
}

intense patient monitoring with emphasis on the respiratory and mental capacities.

Keywords Anesthesia - Obstructive sleep apnea (OSA) . Opioids · Opioid-induced ventilatory impairment (OIVI) . Postoperative pain $\cdot$ Respiratory depression

\section{Introduction}

Obstructive sleep apnea (OSA) is a common health problem; approximately $30 \%$ of the general [1] and surgical [2, 3] population have OSA with most of them lacking formal disease diagnosis [2-5]. Sleep-disordered breathing has been linked to cardiovascular [6, 7] and metabolic [8] morbidity in the general population, and accumulating evidence further suggests that OSA increases the risk for postoperative pulmonary complications $[9,10,11 \cdot 12,13$, $14 \bullet, 15 \bullet \cdot$. Although the causal nature of these complications is unknown, drug-induced ventilatory compromise has been proposed as one of the likely mechanisms [16, 17••].

Several features of the disease suggest that postoperative patients with OSA might be especially vulnerable to opioid side effects such as sedation, diminution of central respiratory drive, and/or increase airway collapsibility. On the other hand, certain physiological components of the disease including nocturnal hypoxemia, sleep disruption, and systemic inflammation, have been associated with either pain processing or opioid analgesia mechanisms. A closer look at these relationships, as well as the variability involved in the compensatory responses to sleep-induced airway obstruction, may allow us to better characterize the risk-versusbenefit ratio regarding the opioid response in the postoperative patient with OSA, and plan better prevention strategies for opioid-related adversities in this population. 


\section{Opioid Analgesia}

Experimental and clinical evidence suggest that two distinct pathophysiological components of OSA, namely sleep disruption and nocturnal intermittent hypoxemia, either acting as sole inflictors or via exciting inflammatory pathways, posses the biologic capacity to enhance pain. Total [18-21], moderate [22••, 23], as well as stageselective [23-25], restriction of sleep have been shown to enhance sensitivity to experimental pain [18, 19, 22••, 23] and increase spontaneous pain complaints in volunteers $[20,21,26 \bullet]$. Furthermore, physiological sleepiness (measured by the maintaining wakefulness test, MWT) predicted increased sensitivity to experimental pain in healthy volunteers [27], while an extended sleep opportunity in sleepy individuals resulted in decreased sleepiness and reduced pain sensitivity [28]. Consistently, insomniacs [29] and temporomandibular joint disorder patients with primary insomnia [30] demonstrated hyperalgesia, while insomnia symptoms [31] and degraded sleep quality [32] predicted daily intensity [32], as well as chronicity [31], of pain in hospitalized burn patients. Although the exact mechanistic details through which disturbed sleep could exacerbate pain remain unclear, experiments on healthy and clinical populations suggest that disrupted sleep may enhance pain behavior either via increasing the expression of hyperalgesic inflammatory mediators $[20,21,33,34 \cdot]$, or by acting on central pain-modulatory networks, i.e., weakening central inhibition and/or reinforcing painfacilitating mechanisms $[22,35,36]$.

In striking contrast with the main finding that sleep disruption enhances pain, temporomandibular joint disorder patients with OSA presented with hypoalgesia to experimental pain [30], suggesting that sleep fragmentation and recurrent nocturnal hypoxemia, both encountered in OSA, may exert different or even opposite effects on pain processing. Even though Smith et al. did not report information on the nocturnal hypoxemia status of these patients, a recent analysis of Cleveland Family Study (CFS; a longitudinal cohort designed to evaluate the familial aggregation of OSA) showed that intermittent hypoxia significantly increased pain reporting in subjects with OSA, independently of the effects of sleep fragmentation and systemic inflammation [37••]. Characteristically, a decrease in the minimum nocturnal arterial saturation $\left(\mathrm{SaO}_{2}\right)$ from 92 to $75 \%$ approximately doubled the odds for experiencing pain. This suggests that the brief periods of hypoxia, which occur repeatedly during sleep in patients with OSA, could either initiate or contribute to enhanced pain experience [38].

While the effect of disturbed sleep on surgical pain and opioid analgesia has yet to be evaluated in the postoperative patient, several lines of evidence support that chronic intermittent hypoxia may increase the sensitivity to the analgesic effect of opioids. In children undergoing adenotonsillectomy, the morphine requirement for postoperative analgesia was inversely associated with the magnitude of preoperative nocturnal recurrent hypoxemia during sleep $[39,40 \bullet \cdot$. These findings were supported by independent experiments showing that intermittent hypoxia upregulated $\mu$-opioid receptors in the developing rat $[41,42]$ and hence it might be responsible for an increased sensitivity to the analgesic and respiratory effects of opioids [43, 44]. In a recent human experiment, both minimum nocturnal $\mathrm{SaO}_{2}$ and insulin growth factor binding protein-1 (IGFBP-1), a serum marker of hypoxia [45], were significantly associated with an increased sensitivity to the opioid analgesic effect $\left[46^{\bullet \bullet}\right]$. In contrast to this evidence, others $[47,48]$ have shown that children with OSA, presented more pain [47], required higher dose of morphine for postoperative analgesia, and experienced prolonged post-anesthetic recovery due to inadequate pain control [48]. In these studies, OSA diagnosis was based upon a polysomnography-determined abnormal apnea/hypopnea index, which might not correlate with either nocturnal hypoxemia or sleep fragmentation phenotypes, making unsafe any inference regarding the possible relationship between nocturnal intermittent hypoxia and postoperative pain and/ or opioid consumption.

Because of the immense variability encountered both in OSA phenotypes $\left[49,50^{\bullet} \cdot\right.$ and pain/analgesia responses in humans, it would require large systematic investigations to fully characterize the effect of OSA on postoperative pain and opioid analgesia in surgical populations. The advantage of identifying the various parameters involved in enhancing pain and/or opioid analgesia in OSA patients is obvious since it could enhance our ability to predict opioid analgesic pharmacology in this population.

\section{Obstructive Apnea Mechanisms}

Current experimental and clinical evidence postulates that OSA is a disorder of ventilatory control [51, 52•]; its severity (as measured by the frequency of apnea/hypopnea events during sleep) is largely determined by the type and effectiveness of compensatory mechanisms that are engaged in response to airway obstruction rather than the anatomical narrowing of the airway, per se [53].

Local anatomical structure and a complex activation pattern of pharyngeal dilator muscles are the primary determinants of upper airway patency during both wakefulness and sleep. Pharyngeal dilators receive input from at least three different types of sources: (a) central respiratory drive (i.e., rising $\mathrm{PaCO}_{2}$ and declining $\mathrm{PaO}_{2}$ ), (b) local negative airway pressure during inspiration (negative 
pressure reflex), and (c) wakefulness drive [52, 54]. In OSA patients, the anatomically compromised airway [55••], compounded with a physiologically diminished pharyngeal dilator activity during sleep (loss of wakefulness drive), undergoes repetitive occlusion, partial or complete, as a result of the negative inspiratory pressure exerted by the diaphragm [56, 57]. Airway obstruction is followed by a gradual and steady rise in the contracting force of pharyngeal dilators as a result of both the negative airway pressure and the rising chemical respiratory drive (i.e., due to rising $\mathrm{PaCO}_{2}$ and declining $\mathrm{PaO}_{2}$ ) with the essential aim of restoring airway patency. Occasionally, arousal from sleep (cortical arousal) would also assist to that end by reinstating the wakefulness drive $[50 \bullet \cdot, 52,58$, 59].

When the rising chemical drive reaches a certain level that can effectively recruit pharyngeal dilators and open the airway (effective recruitment threshold), [50, 60], airway patency is restored. But because of circulatory lung-carotid delays (chemoreceptors sense normal arterial gases with a hysteresis of two to three respiratory cycles), hyperventilation continues (post-obstruction hyperventilation), resulting in hypocapnia and diminished respiratory drive, thus setting the stage for the next obstructive event. Interestingly, in certain cases post-obstruction hyperventilation could be fairly excessive since both intermittent hypoxia and hypercapnia have been shown to promote long-term sensory facilitation of peripheral and central chemoreceptors in humans [61-63] and may thus disproportionately increase the ventilatory response to hypoxic and/or hypercapnic stimuli. Furthermore, cortical arousals, which frequently occur before, at, or even after airway opening, can also promote ventilatory instability via enhancing post-obstruction hyperventilatory response. Although cortical arousals might be the last resort for restoring airway patency and normalize severe hypoxemia associated with certain apnea/hypopnea events, when they occur prematurely (i.e., long before the respiratory drive reaches effective recruitment threshold), would rather precipitate ventilatory instability and promote apneas [64].

Using data from human experiments, Younes [50••] has demonstrated that, depending on the operation of ventilatory control parameters like chemical responsiveness, effective recruitment threshold, and cortical arousal threshold, subjects with identical anatomical airway narrowing of the airway may develop quite different pathophysiological and polysomnographic disease phenotypes, ranging from stable snoring to hypercapnic OSA. The complex dynamics involved, as well as the large interindividual variability that characterizes the control of ventilator responses to obstructive events, make it difficult to predict the exact pattern and frequency of airway obstruction in individual patients and may further complicate effective management of the disease $[65,66]$. For example, whereas therapies like the administration of oxygen [67] and sedatives [68, 69] may benefit patients with increased chemosensory sensitivity and low arousal thresholds, they could be detrimental for others with decreased ventilatory responses to hypoxia/hypercapnia and high arousal thresholds. In the former, oxygen therapy and sedation may act toward stabilizing ventilatory control, whereas in the latter, the same therapeutic measures could prolong the duration of airway obstruction, potentially leading to severe hypoxemia [70, 71]. Although these patients represent a minority among current OSA populations [71], they might be at a greater risk for opioid-related respiratory events in the postoperative period because they rely heavily on arousal to restore adequate airflow and oxygenation; any sedative agent, including opioids, that could further raise arousal thresholds, has the potential to prolong airway obstruction and precipitate hypoxemia. Conversely, in the majority of OSA patients, where frequent obstructive episodes and mild-to-moderate hypoxemia prevail, the sedative effect of opioids may stabilize airway patency and breathing. Interestingly, this contrast between the two main disease phenotypes (i.e., low versus high arousal thresholds associated with the respective pattern of mild versus severe recurrent hypoxemia) is in agreement with the observed juxtaposition between the high prevalence of OSA in surgical populations and the very low incidence of opioid-related critical respiratory events in postoperative patients; i.e., lending support to the hypothesis that only a small subset of OSA patients are at increased risk for opioid-induced respiratory compromise.

\section{Opioid-Induced Ventilatory Impairment}

Opioids interfere with the chemical, behavioral, and motor control of respiration [54, 72]. At analgesic doses, $\mu$-opioid receptor agonists: (a) increase the apneic $\mathrm{PaCO}_{2}$ threshold and suppress ventilatory responses to hypercapnia/hypoxia by acting on the carotid bodies and/or central chemoreceptors located in the brainstem [73••, 74, 75], (b) decrease wakefulness respiratory drive through their action on higher cortical centers [76], and (c) inhibit ventilatory capacity by acting on brainstem motor nuclei, which coordinate the muscular apparatus of breathing, including the diaphragm, chest, and upper airway muscles [77, 78, 79.•]. These side effects of opioids could collectively be labeled as "opioid-induced ventilatory impairment" (OIVI), and result in alveolar hypoventilation with potentially severe hypoxemia, which in rare, unrecognized cases can also be fatal $[17,80]$. Among the various biological elements that could possibly affect the severity of OIVI, comorbidity burden and other factors that are operational in 
the postoperative setting, including pain, residual anesthetic effects, and disturbed sleep may also be modifiers of OIVI [81-84, 85••, 86••, 87].

Retrospective analyses and targeted investigations in large perioperative patient registries suggest that OSA, among other conditions like diabetes [86••], obesity, congestive heart failure, and postoperative renal failure, may present a risk marker for potentially serious or even fatal opioid-related respiratory events that occur in the first $24 \mathrm{~h}$ after surgery $[11 \bullet, 14 \bullet \cdot$ when the effect of opioid analgesics are compounded with the residual effects of general anesthesia $[16,17 \cdot \bullet, 80,88]$. The observation that fatal outcomes were more likely to occur during night time in patients who were difficult to arouse [11•], although not an indicator of direct association with OSA, reinforces the belief that OSA patients with high arousal thresholds, longer obstructive events, and potentially larger desaturations, may have very low reserve to withstand a serious respiratory event in the postoperative period. Admittedly, because of the scarcity of such events $[80,85 \bullet \cdot]$, it is difficult to discern the relative contribution of OSA in the risk for OIVI. Even more so, when obesity, insulin resistance, and diabetes, all frequent comorbidities in patients with OSA [1, 89, 90], have also been associated with altered control of ventilation characterized by a decreased response to hypercapnic/hypoxic challenges, and periodic breathing during sleep [91-93].

Opioids decrease the quantity and alter the architecture of sleep [94•, 95]. Although several other potential factors may disturb sleep in the postoperative patient [96], the effects of opioids may have implications on the severity of OIVI, by modifying the risk-versus-benefit relationship in opioid pharmacology. Opioid analgesics impair basic sleep-wake mechanisms [97••] by inhibiting cholinergic [98, 99], adenosinergic [100, 101], and hypocretinergic [102] transmission. These neurochemical effects of opioids have been causally related to inhibition of rapid eye movement (REM) sleep [103], overall sleep disruption, and decreased sleep consolidation [94] that are implicated in promoting sleepiness and hyperalgesia in humans [104]. As a consequence, opioids, by their effects on sleep-wake mechanisms, have the potential to decrease the safety margin between effective analgesia and ventilatory compromise, especially in patients who are prone to OIVI.

Although the effect of opioids on sleep has not been formally assessed in surgical patients with OSA, the administration of a short-acting opioid during sleep in volunteers with moderate OSA resulted in decreased REM and slow wave sleep, and increased arousals [105•]. Interestingly, the obstructive episodes were decreased, whereas central apneas increased during sleep, a finding probably related to the REM-suppressing effect of the opioid. Theoretically, in certain OSA patients who demonstrate REM-predominant apneas, the obstructive events may recur with increased frequency and severity, during an intense REM sleep rebound after the third postoperative day $[106,107]$ nonetheless the clinical impact of this phenomenon has yet to be shown [17].

Are OSA patients more sensitive to the ventilatory effects of opioids? Current evidence does not support a straightforward answer to this question. To the extent that opioids could further impact the pathophysiology of airway obstruction leading to ventilatory compromise, these agents may precipitate the development of OIVI in the postoperative patient with OSA. However, based on the large variability and dynamics of OSA [50••, 65], it is unlikely that all disease phenotypes share the same risk for OIVI. In certain patients with severe OSA, overlap syndrome (coexistence of OSA with chronic obstructive pulmonary disease), pulmonary arterial hypertension, or obesity hypoventilation syndrome [67, 108], high arousal thresholds and large arterial desaturations, even the slightest opioid-induced suppression of compensatory mechanisms, including their capacity for arousal during airway obstruction, may lead to severe and protracted hypoxemia. Nevertheless, for the typical OSA patient who demonstrates brief obstructive events with mild desaturations and no daytime blood gas abnormalities, the frequent (cortical) arousals from sleep and increased sensitivity to hypoxia and/or hypercapnia, have been described as rather destabilizing factors, potentially aggravating airway obstruction. In these patients, careful titration of opioids to analgesic effect may in fact stabilize airway function by increasing the arousal threshold and dampening chemical responsiveness.

\section{Mitigating Opioid-Related Respiratory Toxicity}

Postoperative hypoxemia is a common side effect of opioids, especially in the immediate postoperative period when patients are still under the residual influence of general anesthetics [85••, 109-111]. Characteristically, in a recent prospective cohort, $15 \%$ of the patients experienced a $\mathrm{SaO}_{2} \leq 85 \%$ for more than a continuous hour during the first $48 \mathrm{~h}$ after elective surgery [112]. Not all patients who present with hypoxemia after surgery have OSA [85, 109111]; on the other hand, not all OSA patients present postoperatively with apneas of increased frequency and nocturnal desaturations of increased severity compared to their preoperative status [113••]. As stated above, based on the high prevalence of OSA among surgical patients [2, 3] in contrast to the very low incidence of respiratory events requiring medical intervention [80, 85*0], OSA diagnosis cannot be considered a reliable risk marker for the latter. Although certain OSA phenotypes might inherently carry a 
risk for drug-induced respiratory compromise, these might be difficult to detect using traditional tests and the development of severe OIVI in the postoperative patient seems to be influenced, among other, largely unknown, parameters, also by age and comorbidity burden. Thus, focusing only on the isolated aspect of sleep-disordered breathing would not be sufficient to predict the overall risk for this complication, even more so when a large fraction of OSA patients remain undiagnosed [114]. Presently, no clinical or laboratory test can outperform clinical judgment in predicting potentially severe respiratory compromise.

Because of the poor risk prediction in this domain, efforts have become more attentive either in minimizing factors that are implicated in precipitating postoperative OIVI, or in detecting the development of OIVI early enough so that an appropriate intervention could prevent an escalation to potentially grave outcomes $[17 \bullet \bullet, 54]$. The use of general anesthetics with short elimination half-life as well as implementation of multimodal analgesic techniques have been proposed to reduce sedation and minimize requirement for opioids in the immediate postoperative period. In the same context, drugs or adjuncts that could counteract opioid-induced respiratory depression without interfering with the analgesic effect of opioids have already been tested in humans with promising results [115-118]. Furthermore, agents that could decrease sedation $[119,120]$ in the postoperative period or provide analgesia without suppressing the function of airway dilator muscles [121] may also be useful adjuncts toward eliminating potentially severe OIVI. Although the development of postoperative hypoxemia in patients with a preoperative diagnosis of OSA cannot be regarded as a surrogate risk marker for severe OIVI, continuous positive airway pressure (CPAP) has been efficacious in reducing hypoxemia related to obstructive events, when applied postoperatively [113••]. Nonetheless, the low patient adherence to CPAP, as well as the ineffectiveness of this therapeutic modality to treat opioid-induced central apneas, may limit its value in preventing serious respiratory events. On the other hand, the use of oxygen in the postoperative patient treated with opioids might also impact our ability to recognize OIVI since a normal $\mathrm{SaO}_{2}$ may mask or enhance [122] central respiratory depression, therefore, obscuring an imminent, potentially severe respiratory event. As noted above, this effect may be especially pronounced in patients with decreased ventilatory responses to hypoxia/ hypercapnia stimuli and high arousal thresholds [67]. Oxygen therapy in those patients might further depress respiration [122] since the latter may be critically dependent on the hypoxic ventilator drive.

Various monitoring modalities, based on objective signals and clinical signs, including arterial oxy-hemoglobin saturation by pulse oximetry, respiratory rate, sedation level, and end-tidal or transcutaneous carbon dioxide monitoring, have been proposed for the early detection of OIVI $[17 \bullet \bullet, 54,87,111]$. However, the efficacy of those methods in eliminating or reducing postoperative OIVI remains to be tested.

\section{Conclusion}

Experimental evidence shows that in patients with obstructive sleep apnea (OSA), intermittent hypoxia and sleep fragmentation may affect opioid pharmacology by enhancing pain and/or sensitivity to opioid analgesia. These effects may have a clinical impact on the determination of risk-versus-benefit ratio for opioid analgesia in the postoperative patient, with emphasis on opioid-induced respiratory toxicity. Ventilatory impairment can be a serious adverse effect of opioids, potentially leading to grave outcomes. Current evidence does not support a direct relationship between preoperative diagnosis of OSA and an increased risk for opioid-induced ventilatory impairment (OIVI) during postoperative analgesic therapy. However, certain pathophysiological features of OSA could be compatible with enhancement of OIVI due to either respiratory compromise or suppression of arousal mechanisms in response to airway obstruction. Because the determinants of OIVI are not limited to factors pertaining to the physiology of sleep-disordered breathing but also include age, comorbidity burden, as well as other, so far unknown, parameters, accurate prediction of potentially severe OIVI in the postoperative patient, remains a challenge. Minimizing perioperative opioid analgesia and postoperative sedation, as well as establishing a close monitoring for postoperative patients, may prove beneficial in preventing OIVI without decreasing the quality of provided analgesia.

\section{Compliance with Ethics Guidelines}

Conflict of Interest Anthony G. Doufas declares that he has no conflict of interest.

Human and Animal Rights and Informed Consent This article does not contain any studies with human or animal subjects performed by any of the authors.

\section{References}

Papers of particular interest, published recently, have been highlighted as:

- Of importance

•- Of major importance

1. Peppard PE, Young T, Barnet JH, Palta M, Hagen EW, Hla KM. Increased prevalence of sleep-disordered breathing in adults. Am J Epidemiol. 2013;177(9):1006-14. 
2. Finkel KJ, Searleman AC, Tymkew H, et al. Prevalence of undiagnosed obstructive sleep apnea among adult surgical patients in an academic medical center. Sleep Med. 2009;10(7):753-8.

3. Singh M, Liao P, Kobah S, Wijeysundera DN, Shapiro C, Chung F. Proportion of surgical patients with undiagnosed obstructive sleep apnoea. Br J Anaesth. 2013;110(4):629-36.

4. Young T, Evans L, Finn L, Palta M. Estimation of the clinically diagnosed proportion of sleep apnea syndrome in middle-aged men and women. Sleep. 1997;20(9):705-6.

5. Memtsoudis SG, Besculides MC, Mazumdar M. A rude awakening - the perioperative sleep apnea epidemic. N Engl J Med. 2013;368(25):2352-3.

6. Peppard PE, Young T, Palta M, Skatrud J. Prospective study of the association between sleep-disordered breathing and hypertension. N Engl J Med. 2000;342(19):1378-84.

7. Yaggi HK, Concato J, Kernan WN, Lichtman JH, Brass LM, Mohsenin V. Obstructive sleep apnea as a risk factor for stroke and death. N Engl J Med. 2005;353(19):2034-41.

8. Punjabi NM, Beamer BA. Alterations in glucose disposal in sleep-disordered breathing. Am J Respir Crit Care Med. 2009;179(3):235-40.

9. Brown KA, Morin I, Hickey C, Manoukian JJ, Nixon GM, Brouillette RT. Urgent adenotonsillectomy: an analysis of risk factors associated with postoperative respiratory morbidity. Anesthesiology. 2003;99(3):586-95.

10. Gali B, Whalen FX, Schroeder DR, Gay PC, Plevak DJ. Identification of patients at risk for postoperative respiratory complications using a preoperative obstructive sleep apnea screening tool and postanesthesia care assessment. Anesthesiology. 2009;110(4):869-77.

11. - Ramachandran SK, Haider N, Saran KA, et al: Life-threatening critical respiratory events: a retrospective study of postoperative patients found unresponsive during analgesic therapy. J Clin Anesth 2011;23(3):207-13. Between 2000 and 2007, 32 among 87,650 patients experienced a postoperative life-threatening critical respiratory event (LT-CRE), while 26 events and three deaths occurred within the first 24 hours of opioid therapy. Four out of 32 patients died. Congestive heart failure, postoperative acute renal failure, obstructive sleep apnea, diabetes mellitus, and hypertension were significantly associated with LT-CREs in adult patients.

12. Gupta RM, Parvizi J, Hanssen AD, Gay PC. Postoperative complications in patients with obstructive sleep apnea syndrome undergoing hip or knee replacement: a case-control study. Mayo Clin Proc. 2001;76(9):897-905.

13. Chung SA, Yuan H, Chung F. A systemic review of obstructive sleep apnea and its implications for anesthesiologists. Anesth Analg. 2008;107(5):1543-63.

14. •- Mokhlesi B, Hovda MD, Vekhter B, Arora VM, Chung F, Meltzer DO. Sleep-disordered breathing and postoperative outcomes after elective surgery: analysis of the Nationwide Inpatient Sample. Chest. 2013;144(3):903-14. In a nationwide retrospective cohort (1,058,710 inpatients between 2004 and 2008) regression analysis estimated significantly higher odds ratio for emergent intubation and mechanical ventilation, noninvasive ventilation, and atrial fibrilation in sleep-disordered breathing $(S D B)$ than in non-SDB patients, after four different types of general surgical procedures. Interestingly, emergent intubations occurred much earlier in the postoperative period in patients with SDB.

15. $\bullet$ Memtsoudis S, Liu SS, Ma Y, et al. Perioperative pulmonary outcomes in patients with sleep apnea after noncardiac surgery. Anesth Analg. 2011;112(1):113-21. The authors retrospectively examined a natiowide cohort of 2,610,441 patients who underwent orthopedic and 3,441,262 patients who underwent general surgical procedures performed between 1998 and 2007. A preoperative diagnosis of sleep apnea (SA) is an independent risk factor for perioperative pulmonary complications like emergent intubation and mechanical ventilation.

16. Ankichetty S, Wong J, Chung F. A systematic review of the effects of sedatives and anesthetics in patients with obstructive sleep apnea. J Anaesthesiol Clin Pharmacol. 2011;27(4):447-58.

17. • Macintyre PE, Loadsman JA, Scott DA. Opioids, ventilation and acute pain management. Anaesth Intensive Care. 2011;39(4):545-58. A detailed introduction of the concept of opioid-induced ventilatory impairment (OIVI) in the setting of acute postoperative pain management. The authors discuss factors that could enhance sensitivity to the central and/or peripheral respiratory depressants effects of opioids, including obstructive sleep apnea and obesity hypoventilation syndromes.

18. Kundermann B, Spernal J, Huber MT, Krieg JC, Lautenbacher S. Sleep deprivation affects thermal pain thresholds but not somatosensory thresholds in healthy volunteers. Psychosom Med. 2004;66(6):932-7.

19. Onen SH, Alloui A, Gross A, Eschallier A, Dubray C. The effects of total sleep deprivation, selective sleep interruption and sleep recovery on pain tolerance thresholds in healthy subjects. J Sleep Res. 2001;10(1):35-42.

20. Haack M, Lee E, Cohen DA, Mullington JM. Activation of the prostaglandin system in response to sleep loss in healthy humans: potential mediator of increased spontaneous pain. Pain. 2009;145(1-2):136-41.

21. Haack M, Sanchez E, Mullington JM. Elevated inflammatory markers in response to prolonged sleep restriction are associated with increased pain experience in healthy volunteers. Sleep. 2007;30(9):1145-52.

22. • Smith MT, Edwards RR, McCann UD, Haythornthwaite JA. The effects of sleep deprivation on pain inhibition and spontaneous pain in women. Sleep. 2007;30(4):494-505. Partial sleep deprivation using the forced-awakening paradigm impaired the function of pain-inhibitory processes and increased spontaneous pain in healthy women.

23. Roehrs T, Hyde M, Blaisdell B, Greenwald M, Roth T. Sleep loss and REM sleep loss are hyperalgesic. Sleep. 2006;29(2):145-51.

24. Moldofsky H, Scarisbrick P. Induction of neurasthenic musculoskeletal pain syndrome by selective sleep stage deprivation. Psychosom Med. 1976;38(1):35-44.

25. Lentz MJ, Landis CA, Rothermel J, Shaver JL. Effects of selective slow wave sleep disruption on musculoskeletal pain and fatigue in middle aged women. J Rheumatol. 1999;26(7):1586-92.

26. - Edwards RR, Almeida DM, Klick B, Haythornthwaite JA, Smith MT. Duration of sleep contributes to next-day pain report in the general population. Pain. 2008;137(1):202-7. In a representative national sample of 971 adults, the authors used structural equation modeling to show that hours of reported sleep on the previous night were highly predictive of the current day's pain frequency. Obtaining less than 6 or more than 9 hours sleep per night was associated with greater pain in the next day.

27. Chhangani BS, Roehrs TA, Harris EJ, et al. Pain sensitivity in sleepy pain-free normals. Sleep. 2009;32(8):1011-7.

28. Roehrs TA, Harris E, Randall S, Roth T. Pain sensitivity and recovery from mild chronic sleep loss. Sleep. 2012;35(12):1667-72.

29. Haack M, Scott-Sutherland J, Santangelo G, Simpson NS, Sethna N, Mullington JM. Pain sensitivity and modulation in primary insomnia. Eur J Pain. 2012;16:522-33.

30. Smith MT, Wickwire EM, Grace EG, et al. Sleep disorders and their association with laboratory pain sensitivity in temporomandibular joint disorder. Sleep. 2009;32(6):779-90. 
31. Smith MT, Klick B, Kozachik S, et al. Sleep onset insomnia symptoms during hospitalization for major burn injury predict chronic pain. Pain. 2008;138(3):497-506.

32. Raymond I, Nielsen TA, Lavigne G, Manzini C, Choiniere M. Quality of sleep and its daily relationship to pain intensity in hospitalized adult burn patients. Pain. 2001;92(3):381-8.

33. Haack M, Pollmacher T, Mullington JM. Diurnal and sleepwake dependent variations of soluble TNF- and IL-2 receptors in healthy volunteers. Brain Behav Immun. 2004;18(4):361-7.

34. - Irwin MR, Wang M, Campomayor CO, Collado-Hidalgo A, Cole S. Sleep deprivation and activation of morning levels of cellular and genomic markers of inflammation. Arch Intern Med. 2006;166(16):1756-62. Partial sleep deprivation (awake from 11 PM to 3 AM) in 30 healthy volunteers was associated with an increase in the expression of interleukin-6 (IL-6) and tumor necrosis factor-alpha $(T N F-\alpha)$ by peripheral blood monocytes.

35. Edwards RR, Grace E, Peterson S, Klick B, Haythornthwaite JA, Smith MT. Sleep continuity and architecture: associations with pain-inhibitory processes in patients with temporomandibular joint disorder. Eur J Pain. 2009;13(10):1043-7.

36. Edwards RR, Quartana PJ, Allen RP, Greenbaum S, Earley CJ, Smith MT. Alterations in pain responses in treated and untreated patients with restless legs syndrome: associations with sleep disruption. Sleep Med. 2011;12(6):603-9.

37. •- Doufas AG, Tian L, Davies MF, Warby SC. Nocturnal intermittent hypoxia is independently associated with pain in subjects suffering from sleep-disordered breathing. Anesthesiology 2013;119(11):1149-62. Intermittent hypoxia significantly increased pain reporting in subjects with OSA, independently of the effects of sleep fragmentation and systemic inflammation; a decrease in the minimum nocturnal arterial saturation $\left(\mathrm{SaO}_{2}\right)$ from 92 to $75 \%$ approximately doubled the odds for experiencing pain.

38. Smith MT, Finan PH. Sleep, respiration, and pain: a potential nexus for chronic pain risk? Anesthesiology. 2013;119(5):1011-3.

39. Brown KA, Laferriere A, Moss IR. Recurrent hypoxemia in young children with obstructive sleep apnea is associated with reduced opioid requirement for analgesia. Anesthesiology. 2004;100(4):806-10.

40. •• Brown KA, Laferriere A, Lakheeram I, Moss IR. Recurrent hypoxemia in children is associated with increased analgesic sensitivity to opiates. Anesthesiology. 2006;105(4):665-69. Preoperative recurrent nocturnal hypoxemia (nadir $\mathrm{SaO}_{2}<85 \%$ versus $\mathrm{SaO}_{2} \geq 85 \%$ ) in children was associated with a $50 \%$ reduction in the morphine requirement for the management of post-adenotonsillectomy pain.

41. Laferriere A, Liu JK, Moss IR. Neurokinin-1 versus mu-opioid receptor binding in rat nucleus tractus solitarius after single and recurrent intermittent hypoxia. Brain Res Bull. 2003;59(4):307-13.

42. Moss IR, Laferriere A. Central neuropeptide systems and respiratory control during development. Respir Physiol Neurobiol. 2002;131(1-2):15-27.

43. Brown KA. Intermittent hypoxia and the practice of anesthesia. Anesthesiology. 2009;110(4):922-7.

44. Moss IR, Brown KA, Laferriere A. Recurrent hypoxia in rats during development increases subsequent respiratory sensitivity to fentanyl. Anesthesiology. 2006;105(4):715-8.

45. Kajimura S, Aida K, Duan C. Insulin-like growth factor-binding protein-1 (IGFBP-1) mediates hypoxia-induced embryonic growth and developmental retardation. Proc Natl Acad Sci USA. 2005;102(4):1240-5.

46. • Doufas AG, Tian L, Padrez KA, et al. Experimental pain and opioid analgesia in volunteers at high risk for obstructive sleep apnea. PLoS One. 2013;8(1):e54807. In adults suffering from obstructive sleep apnea, nocturnal hypoxemia (measured by the nadir arterial saturation during polysomnography) is associated with increased analgesic sensitivity to remifentanil, a shortacting opioid. Insulin growth factor binding protein-1 (IGFBP1), a serum marker of hypoxia, is also associated with increased analgesic potency of remifentanil.

47. Sanders JC, King MA, Mitchell RB, Kelly JP. Perioperative complications of adenotonsillectomy in children with obstructive sleep apnea syndrome. Anesth Analg. 2006;103(5): 1115-21.

48. Sadhasivam S, Chidambaran V, Ngamprasertwong P, et al. Race and unequal burden of perioperative pain and opioid related adverse effects in children. Pediatrics. 2012;129(5):832-8.

49. Xie A, Bedekar A, Skatrud JB, Teodorescu M, Gong Y, Dempsey JA. The heterogeneity of obstructive sleep apnea (predominant obstructive vs pure obstructive apnea). Sleep. 2011;34(6):745-50.

50. $\bullet$ Younes M. Role of respiratory control mechanisms in the pathogenesis of obstructive sleep disorders. J Appl Physiol. 2008;105(5):1389-405. Based on evidence from experimental and clinical studies, the author discusses the role of respiratory control mechanisms in the pathogenesis of obstructive sleep apnea. Apart from the anatomical narrowing of the airway, parameters like the effective recruitment threshold of pharyngeal dilators, the arousal threshold, and the rate of rise of the chemical drive during an obstructive event, determine postobstruction ventilatory overshoot and are major determinants of the polysomnographic features of the disease.

51. Dempsey JA, Veasey SC, Morgan BJ, O’Donnell CP. Pathophysiology of sleep apnea. Physiol Rev. 2010;90(1):47-112.

52. - White DP, Younes MK. Obstructive sleep apnea. Compr Physiol. 2012;2(4):2541-94. An excellent, very detailed review of the most recent evidence and theories regarding the pathophysiology of obstructive sleep apnea.

53. Younes M. Contributions of upper airway mechanics and control mechanisms to severity of obstructive apnea. Am J Respir Crit Care Med. 2003;168(6):645-58.

54. Koo CY, Eikermann M. Respiratory effects of opioids in perioperative medicine. Open Anesthesiol J. 2011;5(Suppl 1-M6):23-34.

55. • Isono S, Remmers JE, Tanaka A, Sho Y, Sato J, Nishino T. Anatomy of pharynx in patients with obstructive sleep apnea and in normal subjects. J Appl Physiol (1985). 1997;82(4):1319-26. The first study to conclusively demonstrate the presence of anatomical abnormalities such as increased narrowing and collapsibility of the passive pharynx in humans suffering from obstructive sleep apnea.

56. Horner RL. Motor control of the pharyngeal musculature and implications for the pathogenesis of obstructive sleep apnea. Sleep. 1996;19(10):827-53.

57. Horner RL, Hughes SW, Malhotra A. State-dependent and reflex drives to the upper airway: basic physiology with clinical implication. J Appl Physiol (1985). 2013;. doi:10.1152/japplphysiol.00531. 2013.

58. Loewen AH, Ostrowski M, Laprairie J, Maturino F, Hanly PJ, Younes M. Response of genioglossus muscle to increasing chemical drive in sleeping obstructive apnea patients. Sleep. 2011;34(8):1061-73.

59. Younes M, Loewen AH, Ostrowski M, Laprairie J, Maturino F, Hanly PJ. Genioglossus activity available via non-arousal mechanisms vs. that required for opening the airway in obstructive apnea patients. J Appl Physiol (1985). 2012;112(2):249-58.

60. Younes M, Ostrowski M, Atkar R, Laprairie J, Siemens A, Hanly P. Mechanisms of breathing instability in patients with obstructive sleep apnea. J Appl Physiol. 2007;103(6): 1929-41.

61. Pialoux V, Hanly PJ, Foster GE, et al. Effects of exposure to intermittent hypoxia on oxidative stress and acute hypoxic ventilatory response in humans. Am J Respir Crit Care Med. 2009;180(10):1002-9. 
62. Babcock M, Shkoukani M, Aboubakr SE, Badr MS. Determinants of long-term facilitation in humans during NREM sleep. J Appl Physiol (1985). 2003;94(1):53-9.

63. Harris DP, Balasubramaniam A, Badr MS, Mateika JH. Longterm facilitation of ventilation and genioglossus muscle activity is evident in the presence of elevated levels of carbon dioxide in awake humans. Am J Physiol Regul Integr Comp Physiol. 2006;291(4):R1111-9.

64. Younes M. Role of arousals in the pathogenesis of obstructive sleep apnea. Am J Respir Crit Care Med. 2004;169(5):623-33.

65. Wang D, Marshall NS, Duffin J, et al. Phenotyping interindividual variability in obstructive sleep apnoea response to temazepam using ventilatory chemoreflexes during wakefulness. J Sleep Res. 2011;20(4):526-32.

66. Smith I, Lasserson TJ, Wright J. Drug therapy for obstructive sleep apnoea in adults. Cochrane Database Syst Rev. 2006;(2): CD003002.

67. Mokhlesi B, Tulaimat A, Parthasarathy S. Oxygen for obesity hypoventilation syndrome: a double-edged sword? Chest. 2011;139(5):975-7.

68. Eckert DJ, Owens RL, Kehlmann GB, et al. Eszopiclone increases the respiratory arousal threshold and lowers the apnoea/hypopnoea index in obstructive sleep apnoea patients with a low arousal threshold. Clin Sci (Lond). 2011;120(12): 505-14.

69. Eikermann M, Fassbender P, Zaremba S, et al. Pentobarbital dose-dependently increases respiratory genioglossus muscle activity while impairing diaphragmatic function in anesthetized rats. Anesthesiology. 2009;110(6):1327-34.

70. Hudgel DW, Hendricks C, Dadley A. Alteration in obstructive apnea pattern induced by changes in oxygen- and carbon-dioxide-inspired concentrations. Am Rev Respir Dis. 1988;138(1): $16-9$.

71. Eckert DJ, Younes MK. Arousal from sleep: implications for obstructive sleep apnea pathogenesis and treatment. J Appl Physiol (1985). 2013; doi:10.1152/japplphysiol.00649.2013.

72. Stuth EA, Stucke AG, Zuperku EJ. Effects of anesthetics, sedatives, and opioids on ventilatory control. Compr Physiol. 2012;2(4):2281-367.

73. • Dahan A, Romberg R, Teppema L, Sarton E, Bijl H, Olofsen E. Simultaneous measurement and integrated analysis of analgesia and respiration after an intravenous morphine infusion. Anesthesiology. 2004;101(5):1201-9. First study to demonstrate in humans that systems involved in the analgesic and respiratory-depressant effects of opioids share important pharmacodynamic characteristics like similar onset/offset times and similar sensitivities to morphine.

74. Weil JV, McCullough RE, Kline JS, Sodal IE. Diminished ventilatory response to hypoxia and hypercapnia after morphine in normal man. N Engl J Med. 1975;292(21):1103-6.

75. Berkenbosch A, Teppema LJ, Olievier CN, Dahan A. Influences of morphine on the ventilatory response to isocapnic hypoxia. Anesthesiology. 1997;86(6):1342-9.

76. Pattinson KT, Governo RJ, MacIntosh BJ, et al. Opioids depress cortical centers responsible for the volitional control of respiration. J Neurosci. 2009;29(25):8177-86.

77. McKay LC, Feldman JL. Unilateral ablation of pre-Botzinger complex disrupts breathing during sleep but not wakefulness. Am J Respir Crit Care Med. 2008;178(1):89-95.

78. McKay LC, Janczewski WA, Feldman JL. Sleep-disordered breathing after targeted ablation of preBotzinger complex neurons. Nat Neurosci. 2005;8(9):1142-4.

79. • Montandon G, Qin W, Liu H, Ren J, Greer JJ, Horner RL. PreBotzinger complex neurokinin-1 receptor-expressing neurons mediate opioid-induced respiratory depression. J Neurosci. 2011;31(4):1292-301. This experimental work establishes in adult rats that neurokinin-1 receptor-expressing neurons in the preBötzinger complex of medulla are responsible for mediating the respiratory depressant effect (i.e., decrease in respiratory rate) of opioids.

80. Dahan A, Aarts L, Smith TW. Incidence, reversal, and prevention of opioid-induced respiratory depression. Anesthesiology. 2010;112(1):226-38.

81. Hillman DR, Platt PR, Eastwood PR. The upper airway during anaesthesia. Br J Anaesth. 2003;91(1):31-9.

82. Borgbjerg FM, Nielsen K, Franks J. Experimental pain stimulates respiration and attenuates morphine-induced respiratory depression: a controlled study in human volunteers. Pain. 1996;64(1):123-8.

83. Sarton E, Dahan A, Teppema L, et al. Acute pain and central nervous system arousal do not restore impaired hypoxic ventilatory response during sevoflurane sedation. Anesthesiology. 1996;85(2):295-303.

84. Karan S, Voter W, Palmer L, Ward DS. Effects of pain and audiovisual stimulation on the opioid-induced depression of the hypoxic ventilatory response. Anesthesiology. 2005;103(2): 384-90.

85. - Cashman JN, Dolin SJ. Respiratory and haemodynamic effects of acute postoperative pain management: evidence from published data. Br J Anaesth. 2004;93(2):212-23. The authors reviewed a total of 165 published investigations (nearly 20,000 patients) providing evidence for opioid-related adverse effects in the context of postoperative pain management. Across different analgesic techniques, the incidence of opioid-induced respiratory depression defined by low respiratory rate was less than $1 \%$.

86. $\bullet$ Rose DK, Cohen MM, Wigglesworth DF, DeBoer DP. Critical respiratory events in the postanesthesia care unit: patient, surgical, and anesthetic factors. Anesthesiology. 1994;81(2): 410-18. The authors prospectively collected data in 24,157 consecutive surgical patients receiving general anesthesia with the scope of identifying critical respiratory events (CRE) during the immediate postanesthetic recovery period. The overall risk of a CRE comprised by hypoxemia $\left(\mathrm{SaO}_{2}<90 \%\right.$ : $\left.0.9 \%\right)$, hypoventilation (respiratory rate $<8$ breaths $/$ min: $0.2 \%$ ), and airway obstruction (stridor or laryngospasm: $0.2 \%$ ) was estimated at $1.3 \%$.

87. Overdyk FJ, Hillman DR. Opioid modeling of central respiratory drive must take upper airway obstruction into account. Anesthesiology. 2011;114(1):219-20.

88. Niesters M, Overdyk F, Smith T, Aarts L, Dahan A. Opioidinduced respiratory depression in paediatrics: a review of case reports. Br J Anaesth. 2013;110(2):175-82.

89. Ip MS, Lam B, Ng MM, Lam WK, Tsang KW, Lam KS. Obstructive sleep apnea is independently associated with insulin resistance. Am J Respir Crit Care Med. 2002;165(5):670-6.

90. Liu A, Kushida CA, Reaven GM. Risk for obstructive sleep apnea in obese, nondiabetic adults varies with insulin resistance status. Sleep Breath. 2013;17(1):333-8.

91. Mancini M, Filippelli M, Seghieri G, et al. Respiratory muscle function and hypoxic ventilatory control in patients with type I diabetes. Chest. 1999;115(6):1553-62.

92. Resnick HE, Redline S, Shahar E, et al. Diabetes and sleep disturbances: findings from the Sleep Heart Health Study. Diabetes Care. 2003;26(3):702-9.

93. Weisbrod CJ, Eastwood PR, O'Driscoll G, Green DJ. Abnormal ventilatory responses to hypoxia in Type 2 diabetes. Diabet Med. 2005;22(5):563-8.

94. - Cronin AJ, Keifer JC, Davies MF, King TS, Bixler EO. Postoperative sleep disturbance: influences of opioids and pain in humans. Sleep. 2001;24(1):39-44. Postoperative sleep disturbance, characterized by a great reduction in slow wave and 
rapid eye movement sleep, were affected by neither adequate pain control nor elimination of the use of opioids.

95. Krenk L, Jennum P, Kehlet H. Sleep disturbances after fast-track hip and knee arthroplasty. Br J Anaesth. 2012;109(5):769-75.

96. Rosenberg-Adamsen S, Kehlet H, Dodds C, Rosenberg J. Postoperative sleep disturbances: mechanisms and clinical implications. Br J Anaesth. 1996;76(4):552-9.

97. •- Lydic R, Baghdoyan HA. Sleep, anesthesiology, and the neurobiology of arousal state control. Anesthesiology. 2005;103(6):1268-95. A detailed review about the cellular and molecular mechanisms contributing to arousal state control with special reference to the action of anesthetics and opioids.

98. Lydic R, Keifer JC, Baghdoyan HA, Becker L. Microdialysis of the pontine reticular formation reveals inhibition of acetylcholine release by morphine. Anesthesiology. 1993;79(5):1003-12.

99. Mortazavi S, Thompson J, Baghdoyan HA, Lydic R. Fentanyl and morphine, but not remifentanil, inhibit acetylcholine release in pontine regions modulating arousal. Anesthesiology. 1999;90(4):1070-7.

100. Nelson AM, Battersby AS, Baghdoyan HA, Lydic R. Opioidinduced decreases in rat brain adenosine levels are reversed by inhibiting adenosine deaminase. Anesthesiology. 2009;111(6): 1327-33.

101. Wu M, Sahbaie P, Zheng M, et al. Opiate-induced changes in brain adenosine levels and narcotic drug responses. Neuroscience. 2013;228:235-42.

102. Li Y, van den Pol AN. Mu-opioid receptor-mediated depression of the hypothalamic hypocretin/orexin arousal system. J Neurosci. 2008;28(11):2814-9.

103. Cronin A, Keifer JC, Baghdoyan HA, Lydic R. Opioid inhibition of rapid eye movement sleep by a specific mu receptor agonist. Br J Anaesth. 1995;74(2):188-92.

104. Moore JT, Kelz MB. Opiates, sleep, and pain: the adenosinergic link. Anesthesiology. 2009;111(6):1175-6.

105. Bernards CM, Knowlton SL, Schmidt DF, et al. Respiratory and sleep effects of remifentanil in volunteers with moderate obstructive sleep apnea. Anesthesiology. 2009;110(1):41-49. Administration of remifentanil in subjects suffering from moderate obstructive sleep apnea (OSA) decreased obstructive and increased central apneas, likely due to the marked reduction in rapid eye movement sleep. As a consequence, remifentanil administration also decreased arterial oxy-hemoglobin saturation.

106. Knill RL, Moote CA, Skinner MI, Rose EA. Anesthesia with abdominal surgery leads to intense REM sleep during the first postoperative week. Anesthesiology. 1990;73(1):52-61.

107. Rosenberg J, Wildschiodtz G, Pedersen MH, von Jessen F, Kehlet H. Late postoperative nocturnal episodic hypoxaemia and associated sleep pattern. Br J Anaesth. 1994;72(2):145-50.

108. Chau EH, Lam D, Wong J, Mokhlesi B, Chung F. Obesity hypoventilation syndrome: a review of epidemiology, pathophysiology, and perioperative considerations. Anesthesiology. 2012;117(1):188-205.
109. Bowdle TA. Nocturnal arterial oxygen desaturation and episodic airway obstruction after ambulatory surgery. Anesth Analg. 2004;99(1):70-6.

110. Catley DM, Thornton C, Jordan C, Lehane JR, Royston D, Jones JG. Pronounced, episodic oxygen desaturation in the postoperative period: its association with ventilatory pattern and analgesic regimen. Anesthesiology. 1985;63(1):20-8.

111. Overdyk FJ, Carter R, Maddox RR, Callura J, Herrin AE, Henriquez C. Continuous oximetry/capnometry monitoring reveals frequent desaturation and bradypnea during patientcontrolled analgesia. Anesth Analg. 2007;105(2):412-8.

112. Shahinyan A, Sun Z, Finnegan PS, et al. Duration and incidence of postoperative hypoxemia. In: Presented at the annual meeting of the American Society of Anesthesiologists. 2013;119:BOC12.

113. • Liao P, Luo Q, Elsaid H, Kang W, Shapiro CM, Chung F. Perioperative auto-titrated continuous positive airway pressure treatment in surgical patients with obstructive sleep apnea: a randomized controlled trial. Anesthesiology. 2013;119(4):837-47. The postoperative application of continuous positive airway pressure (CPAP) decreased the frequency of apnea/hyponea events and improved nocturnal oxygenation in surgical patients with obstructive sleep apnea. However, patients' adherence to CPAP was less than $50 \%$.

114. Kaw R, Michota F, Jaffer A, Ghamande S, Auckley D, Golish J. Unrecognized sleep apnea in the surgical patient: implications for the perioperative setting. Chest. 2006;129(1):198-205.

115. Oertel BG, Felden L, Tran PV, et al. Selective antagonism of opioid-induced ventilatory depression by an ampakine molecule in humans without loss of opioid analgesia. Clin Pharmacol Ther. 2010;87(2):204-11.

116. Dahan A, Roozekrans M, van der Schrier R, Smith T, Aarts L. Primum non nocere or how to resolve drug-induced respiratory depression. Anesthesiology. 2013;118(6):1261-3.

117. Teppema LJ, Bijl H, Romberg RR, Dahan A. Antioxidants reverse depression of the hypoxic ventilatory response by acetazolamide in man. J Physiol. 2006;572(Pt 3):849-56.

118. Teppema LJ, Romberg RR, Dahan A. Antioxidants reverse reduction of the human hypoxic ventilatory response by subanesthetic isoflurane. Anesthesiology. 2005;102(4):747-53.

119. Meuret P, Backman SB, Bonhomme V, Plourde G, Fiset P. Physostigmine reverses propofol-induced unconsciousness and attenuation of the auditory steady state response and bispectral index in human volunteers. Anesthesiology. 2000;93(3):708-17.

120. Hedner J, Kraiczi H, Peker Y, Murphy P. Reduction of sleepdisordered breathing after physostigmine. Am J Respir Crit Care Med. 2003;168(10):1246-51.

121. Eikermann M, Grosse-Sundrup M, Zaremba S, et al. Ketamine activates breathing and abolishes the coupling between loss of consciousness and upper airway dilator muscle dysfunction. Anesthesiology. 2012;116(1):35-46.

122. Niesters M, Mahajan RP, Aarts L, Dahan A. High-inspired oxygen concentration further impairs opioid-induced respiratory depression. Br J Anaesth. 2013;110(5):837-41. 